\title{
BioAssay templates for the semantic web
}

Alex M Clark, Nadia K Litterman, Janice E Kranz, Peter Gund, Kellan Gregory, Barry A Bunin

Annotation of bioassay protocols using semantic web vocabulary is a way to make experiment descriptions machine-readable. Protocols are communicated using concise scientific English, which precludes most kinds of analysis by software algorithms. Given the availability of a sufficiently expressive ontology, some or all of the pertinent information can be captured by asserting a series of facts, expressed as semantic web triples (subject, predicate, object). With appropriate annotation, assays can be searched, clustered, tagged and evaluated in a multitude of ways, analogous to other segments of drug discovery informatics. The BioAssay Ontology (BAO) has been previously designed for this express purpose, and provides a layered hierarchy of meaningful terms which can be linked to. Currently the biggest challenge is the issue of content creation: scientists cannot be expected to use the BAO effectively without having access to software tools that make it straightforward to use the vocabulary in a canonical way. We have sought to remove this barrier by: (1) defining a bioassay template data model; (2) creating a software tool for experts to create or modify templates to suit their needs; and (3) designing a common assay template (CAT) to leverage the most value from the BAO terms. The CAT was carefully assembled by biologists in order to find a balance between the maximum amount of information captured vs. low degrees of freedom in order to keep the user experience as simple as possible. The data format that we use for describing templates and corresponding annotations is the native format of the semantic web (RDF triples), and we demonstrate some of the ways that generated content can be meaningfully queried using the SPARQL language. We have made all of these materials available as open source (http://github.com/cdd/bioassay-template), in order to encourage community input and use within diverse projects, including but not limited to our own commercial electronic lab notebook products. 
1 BioAssay Templates for the Semantic Web

2

3 Alex M. Clark (corresponding author)

4 Nadia K. Litterman

5 Janice E. Kranz

6 Peter Gund

7 Kellan Gregory

8 Barry A. Bunin

9

Abstract

Annotation of bioassay protocols using semantic web vocabulary is a way to make experiment descriptions machine-readable. Protocols are communicated using concise scientific English, which precludes most kinds of analysis by software algorithms. Given the availability of a sufficiently expressive ontology, some or all of the pertinent information can be captured by asserting a series of facts, expressed as semantic web triples (subject, predicate, object). With appropriate annotation, assays can be searched, clustered, tagged and evaluated in a multitude of ways, analogous to other segments of drug discovery informatics. The BioAssay Ontology (BAO) has been previously designed for this express purpose, and provides a layered hierarchy of meaningful terms which can be linked to. Currently the biggest challenge is the issue of content creation: scientists cannot be expected to use the BAO effectively without having access to software tools that make it straightforward to use the vocabulary in a canonical way. We have sought to remove this barrier by: (1) defining a bioassay template data model; (2) creating a software tool for experts to create or modify templates to suit their needs; and (3) designing a common assay template (CAT) to leverage the most value from the BAO terms. The CAT was carefully assembled by biologists in order to find a balance between the maximum amount of information captured vs. low degrees of freedom in order to keep the user experience as simple as possible. The data format that we use for describing templates and corresponding annotations is the native format of the semantic web (RDF triples), and we demonstrate some of the ways that generated content can be meaningfully queried using the SPARQL language. We have made all of these materials available as open source (http://github.com/cdd/bioassay-template), in order to encourage community input and use within diverse projects, including but not limited to our own commercial electronic lab notebook products.

\section{Introduction}

One of the major problems currently being faced by biologists charged with the task of performing experimental assays on pharmaceutically interesting molecules is the information burden involved with handling collections of assay descriptions. Individual laboratories may carry out hundreds or even thousands of screening experiments each year. Each of these experiments involves a protocol, and any two experiments may be identical, similar, or completely different. The typical practice for describing bioassay protocols, for both external communication and internal record keeping, is to use concise scientific English, which is the 
42 most universally human readable method of communication, assuming the recipient is familiar

43 with the relevant jargon.

44 Unfortunately this method is not scalable. Even given the availability of an expert, it is often

45 quite difficult and time-consuming to read two assay description paragraphs and provide a metric for the degree to which two protocols differ. There are many workflow scenarios where comparison of protocols is necessary, e.g. searching through a collection of previous experiments, or making a judgment call as to whether two batches of small molecule measurements are comparable. Attempting to use software to assist with such tasks, when the substrate is unconstrained text, results in solutions that are crude at best.

While these issues with scalability could be described as a relatively minor nuisance in a small laboratory, the field of drug discovery has lately been undergoing a renaissance of open data. ${ }^{1,2}$, ${ }^{3,4}$ Services such as PubChem provide a truly massive resource $;{ }^{5}$ PubChem alone provides more than a million unique bioassay descriptions, and is growing rapidly. ${ }^{6,7}$ Such data are supplemented by carefully curated resources like ChEMBL, ${ }^{8}$ which are much smaller but have strict quality control mechanisms in place. What these services have in common is that their bioassay protocols have very little machine-readable content. In many cases, information about the target, and the kind and units of the measurements, have been abstracted out and represented in a marked up format, but all of the remaining particulars of the protocol are ensconced within English grammar, if at all.

In order to address this problem, the BioAssay Ontology (BAO) was devised.9,10,11 The BAO, which includes relevant components from other ontologies, is a semantic web vocabulary that contains thousands of terms for biological assay screening concepts, arranged in a series of layered class hierarchies. The BAO is extensive and detailed, and easily extensible. The vocabulary is sufficiently expressive to be used for describing biological assays in a systematic way, yet it has seen limited use. Influential projects such as PubChem, ${ }^{12}$ ChEMBL, ${ }^{13}$ BARD ${ }^{14}$ and OpenPHACTS ${ }^{15}$ make use of the ontology, but the level of description in each is shallow, using only a small fraction of the terms.

There are a number of factors holding back scientists from using the BAO and related ontologies to describe their assays in detail, with perhaps the most substantial being the lack of software that makes the annotation process fast and convenient. Because it is based on the semantic web, BAO concepts are expressed as triples, of the form [subject, predicate, object]. There are no hard rules about how this is applied, which is a characteristic of the semantic web, and is both an asset and a liability. The simplest way to consider annotating a particular feature of an assay, e.g. the biological process, is to compose a triple of a form such as [assay ID, biological process, viral genome replication]. Each of these 3 fields is a uniform resource indicator (URI), which points to a globally unique object with established meaning. In this case, assay ID would correspond to an identifier that the user has created for the assay description; biological process corresponds to a specific property in the BAO that is used to link assays and the biological process that is being affected; and viral genome replication refers to a class in the $\mathrm{BAO}$, which identifies a specific instance of a biological process, which is in turn inherited from a sequence of increasingly general classes, and may also be linked to any other node within the greater semantic web, such as the extensive Gene Ontology (GO) ${ }^{16}$.

In principle, screening biologists can use the properties and classes from the BAO to annotate their assays intelligently in a machine readable format that is compatible with the universe of the semantic web. If large numbers of assays were sufficiently annotated, biologists and other drug discovery scientists could perform advanced searches and filtering that would enable better interpretation of results, enhanced building of machine-learning models, and uncovering of experimental artifacts. Despite the clear benefits of semantic annotation, the BAO remains 
90 largely unused, the primary reason being its lack of accessibility. The BAO and its linked

91 dependencies are large, and can be expected to keep growing as they are extended to capture

92 more biological concepts. For an interactive view onto these terms, the site

http://bioportal.bioontology.org/ontologies/BAO should be used to peruse the hierarchy. ${ }^{17}$ Figure 1 shows two snapshots of part of the BAO hierarchy, using the BioPortal resource. The classes (Figure 1a) that make up the ontology contain the bulk of the terms and provide most of the expressive value, while the properties (Figure 1b) are used to provide context. The class hierarchy is in places many levels deep, and although it is arranged in a logical pattern, it is nonetheless necessary to be familiar with the entire layout in order to meaningfully annotate an assay protocol. Even an expert biologist familiar with the entire ontology would be presented with multiple degrees of freedom for deciding how to annotate a protocol; this is a fundamental problem for machine readability, which requires uniform consistency.

In our previous work we addressed the end-user problem, and invented technology that applies to the scenario when a user is presented with plain English text, and is charged with the task of selecting the appropriate semantic annotations. Our solution involved a hybrid approach that combined natural language processing with machine learning based on training data, with an intuitive interface that helps the user select the correct annotations, leaving the final choice in the hands of the scientist. ${ }^{18}$ During this process we found that the challenge that we were unable to fully overcome was the burden of creating new training data. The BAO vocabulary defines more than 2500 classes, in addition to properties and terms from other ontologies, all of which can be expected to grow as the BAO is increasingly used for more biological content.

111 Considering each term as it applies to a given assay requires a high level of expertise of the

112 BAO itself. For example, the NIH's Molecular Libraries Program's bioassay database, known as

113 the BARD, employed dedicated research staff to annotate more than two thousand assays. ${ }^{19}$

114 The absence of clear and straightforward guidance as to which terms to use under what circumstances is preventing adoption of the BAO by drug discovery scientists. For our model building efforts, we made use of a training data set made up of 1066 PubChem bioassays that each had more than a hundred terms associated with them, ${ }^{20,21}$ although not all of the annotations were able to be matched to ontology terms. For purposes of creating additional training data, we experienced considerable difficulty finding what we considered to be canonical annotations for any given assay.

121 The BAO is essentially a vocabulary that is capable of describing many assay properties, but it 122 lacks instructions on its use. This is an issue that we have undertaken to solve, and in this 123 article we describe our approach to providing this critical missing component.

124 We describe a data model called the BioAssay Template (BAT), which consists of a small number of terms which are organized to describe how the BAO and linked ontologies should be used to describe a particular kind of bioassay. A template is essentially a gateway to the overall ontology, which divides the assay annotation process into a fixed hierarchy of assignments, each of which has a prescribed list of values, which are cherry-picked from the overall ontology.

129 The BAT vocabulary can be used to create any number of templates, which can be customized

130 to suit the task at hand. As a starting point, we have created what we refer to as the common assay template (CAT). CAT is an annotation recipe that is intended to capture the major properties that most biologists need to describe their assays and that enables most drug

133 discovery scientists to have a basic understanding of an assay and its results.

134 A condensed summary of this template is shown in Figure 2. Unlike the class hierarchy of the $135 \mathrm{BAO}$, the tree structure of the CAT is flat. While the data model allows groups and subgroups, 136 our current template errs on the side of simplicity, and includes just 16 different assignments, 
137 each of which is associated directly with the top-level assay, and each of which has a list of

138 associated values (examples shown in Figure 2).

139 A template can be customized as necessary, and once it is ready, it can be used to define the

140 way in which assays are annotated. The data model is designed to enable software to compose

141 a user interface: presenting each of the categories, and making use of the selected values as

142 the options that are made available to the user. It is essentially a way to restrict and simplify the

143 large scope of the BAO, reduce the degrees of freedom, and remove ambiguity. Having curated

144 the assignments and values so that the lists consist of the minimum number of relevant

145 possibilities, each of them decorated by a meaningful label and a more detailed description, it

146 becomes possible to design a user experience that is suitable for a scientist who is an expert in

147 the field, but does not necessarily know anything about semantic web concepts.

148 In order to explore this approach, we have created a software package called the BioAssay

149 Schema Editor, which is open source and available via GitHub. It is written using Java 8, and

150 runs on the major desktop platforms (Windows, Mac \& Linux). The software implements the

151 data model that we describe in this article.

152 Our priorities for this work are to: (1) establish a data model for bioassay templates; (2) create

153 an intuitive software package for editing these templates and using them to annotate real data;

154 and (3) collaboratively establish a CAT for general purpose use. We have put a considerable

155 amount of effort into the user interface for editing templates, even though we expect only a

156 small fraction of biologists will ever be directly involved in editing them. We have also invested

157 significant effort towards developing a one-size-fits-most template, the CAT. Our goal with the

158 CAT was to enable capture of $\sim 80 \%$ of the most commonly used terms, and present them in a

159 logical and concise way, so that a large proportion of users will be able to use it as-is to add a

160 significant amount of value to their protocol data. In addition, the CAT can act as a starting point

161 for modification if scientists would like to tailor the template.

162 Scientists working in research groups that routinely make use of terms that are not included in

163 the CAT can elect to start with an existing template and add the missing assignments and

164 values, and also delete whole groups of content that do not apply to their research. A research

165 group may accumulate a collection of task-specific templates, allowing their scientists to pick the

166 most appropriate one. By ensuring that the editor software is easy to use, runs on all platforms,

167 and is open source, we hope to ensure that this option is quite practical for any research group

168 with access to basic information technology expertise. We intend to encourage the community

169 to make use of these resources, both as standalone tools and interoperating with the electronic

170 lab notebook software that we are presently designing.

171 One of the implicit advantages of using semantic web technology as the underlying data format

172 (triples), and a well established set of reference terms (the BAO and various linked ontologies),

173 is that even if two scientists are annotating assays with different templates, it is highly likely that

174 many or most of the terms will overlap, even if the templates were created from scratch. Since

175 the final deliverable for an annotated assay is the semantic web, it means that the output can be

176 subjected to the entire universe of software designed to work with RDF triple stores. ${ }^{22}$ As more

177 assays are annotated, the scope and power of queries and informatics approaches for

178 enhancing drug discovery projects are similarly increased. With a large corpus of annotated

179 assays available, scientists will be able to make better use of prior work for understanding

180 structure activity relationships, uncovering experimental artifacts, building machine-learning

181 models, and reducing duplicated efforts. 
182 Methods

\section{Data Model}

184 The semantic description of templates and annotations uses a small number of additional URIs, each of which has the root stem http://bioassayontology.org/bat, and is denoted using the Turtle-style ${ }^{23}$ abbreviated prefix "bat".

187 The hierarchical model for describing a template is shown in Figure 3. Parent:child relationships denoted by an arrow indicate one-to-many relationships, while the properties listed in the boxes underneath the nodes are one-to-one relationships. A template definition begins with the root, which is distinguished by being of type bat:BioAssayTemplate. The root is also of type bat:Group, and has some number of child nodes, which are themselves either assignments or subgroups.

An assignment node has several scalar properties, including label and description, and it also refers to a property resource. These are typically mapped to URI resources found within the BAO (e.g. http://www.bioassayontology.org/bao\#BAO_0000205, label: "has assay format"). Each assignment has some number of values associated with it, and these make up the list of available options. Each value is primarily identified by the resource that it maps to, which is typically found in the BAO (e.g. http://www.bioassayontology.org/bao\#BAO_0000219, label: "cell based format"). Besides the label and description, which are customizable within the template data model, the reference URI has its own implied class hierarchy (e.g. "cell based format" is a subclass of "assay format"), which is not encoded in the template data model, but is inferred once it is paired with the BAO and its linked ontologies.

The schema for annotation of assays is shown in Figure 4. The assay is given a distinct URI, and is associated with several properties such as label and description. The template is recorded, as is an optional reference to the origin of the assay (which may be a semantic web resource, or a DOI link to a journal article). The free-text description of the assay can also be recorded using the hasParagraph predicate.

The assay is associated with some number of annotations, which are primarily linked to assignments within the corresponding template. For annotations that assert a URI link, the hasValue predicate typically corresponds to one of the available values that was prescribed for the assignment in the template definition, and generally refers to a term defined in the BAO, though custom references can be used - or the annotation may be specified using the hasLiteral predicate instead, which means that the user has entered data in a different form, typically text or a numeric value. The hasProperty predicate is generally copied from the corresponding assignment.

When annotating an assay, each assignment may be used any number of times, i.e. zero instances means that it has been left blank, while asserting two or more triples means that all of the values apply. The relationship between assays and annotations has no nesting: the intrinsic group/sub-group structure of any particular annotation can be inferred from the template, since the usesTemplate and isAssignment predicates refer to the origins in the template.

\section{Software}

222 The BioAssay Schema Editor is available from GitHub (https://github.com/cdd/bioassay223 template) and may be used under the terms of the Gnu Public License 2.0. ${ }^{24}$ The code is written using Java 8, and the user interface is based on JavaFX. Semantic web functionality is implemented by incorporating the Apache Jena library. ${ }^{25}$ The project includes a snapshot of the BioAssay Ontology ${ }^{26}$ and some of the linked ontologies, as well as the latest version of the 
227

228

229

230

231

232

233

234

235

236

237

238

239

240

241

242

243

244

245

246

247

248

249

250

251

252

253

254

255

256

257

258

259

260

261

262

263

264

265

266

267

268

269

270 common assay template schema. It should be assumed that the project will continue to evolve until well after the publication date of this article.

The application operates on a datafile referred to as a schema, which is represented as a collection of triples (in Turtle format, with the extension .ttl). A schema is expected to include a single template, for which the root node is of type bat:BioAssayTemplate, and may optionally contain any number of assays that have been (or will be) annotated using that same template. Triples are used as the serialization format in order that the editable files can be used as-is by a Triple store, and become a part of the semantic web with no further modification.

Figure 5 shows the main window for the application, which has loaded a contemporary version of the common assay template (CAT), and has several accompanying assays awaiting annotation. The components that make up the template are shown as a hierarchy on the left hand side of the panel. Selecting any of the groups or assignments causes the detail view on the right to be filled in with the corresponding content.

Adding, deleting, renaming etc. of groups, assignments and values is fairly mundane, and follows standard desktop user interface design patterns. Selecting URI values for properties and values requires a more specific interface, and is composed by summarizing the BAO vocabulary, which is loaded into the application at the beginning. Resources can be selected using a dialog box that can present the list of options in a flat list, with an optional search box for restricting the list (Figure 6a) or by using the hierarchy view that shows the position in the BAO ontology (Figure 6b). The dialog box can also be used to add multiple values at once, which is particularly convenient when a branch of the BAO encompasses multiple terms that are all valid options. When a resource is selected, its label and description are imported from the BAO into the template: these values can be edited after the fact, but by default they are the same as in the underlying vocabulary.

The primary role of the schema editor is to provide a convenient way to edit templates, but in support of this goal, it also provides an interface to use the template to annotate assays. The interface can be used for generating training data (e.g. for model generation), but it is mainly intended as a way to 'test drive' the current template. Because the annotation process is directly derived from the template, having the two editing processes side by side is advantageous when the template is being designed. For example, the operator can begin annotating an assay, and if a value is missing from one of the assignments, or a new kind of assignment turns out to be necessary, this can be added to the template within the same editing session.

Figure 7a shows an example of an assay that has been annotated. The detail view has a placeholder for description text, which is particularly useful when the content has been imported from some external source, and the annotations are being made by converting the protocol text into semantic annotations. Clicking on any of the annotation buttons brings up a panel of options (Figure 7b) that represent the prescribed values for the assignment. Each of the assignments can be left blank, annotated once, or given multiple values. The ideal use case is when the value (or values) occurs within the list of prescribed values, but since the data model allows any URI, the user interface also allows the user to insert a custom URI. In cases where no URI is listed in the template (e.g. a concept that does not have an established URI), it is possible to add plain text for any of the assignment annotations. While this has no meaning from a machine-learning point of view, it can serve as a convenient placeholder for terms that will be invented in the future. 


\section{Results}

\section{Templates}

273 We set out to create a common assay template (CAT) that includes the basic details essential to defining any bioassay: assay type, format, target and biology, results and pharmacology, and other details. The CAT was developed with the opposing goals of identifying assignments that (1) would be limited in number in order to be not overly burdensome vs. (2) comprehensively cover the majority of the information contained in written descriptions of bioassays. We also considered the type of information that would be utilized by an end user attempting to search, filter, and aggregate assays by their bioassay annotations. For example, details such as the assay footprint (plate type), assay kit, and detection instrument were included because they may be useful terms for identifying experimental artifacts. Biological process and other targetrelated information were included to enable aggregating results across similar drug discovery projects for model-building and other applications. Finally, we limited assignments to those where the BAO offered sufficient options for possible values. Since the goal of the project is to generate machine-readable assay annotations, we avoided assignments where BAO terms were not available, such as those characterizing in vivo assays, and especially assignments whose values would be very specific for each assay, such as negative and positive controls. These areas will be addressed in the future once the underlying vocabulary (BAO or otherwise) is available sufficient to expand the domain. Similarly, the CAT falls short of capturing detailed protocol steps. In its present incarnation, it cannot be considered as a complete replacement for the text that is typically used to describe an assay, though we do intend to pursue this level of detail in future work. For the present, we are primarily concerned with utilizing the rich vocabulary within the BAO to achieve maximum impact with minimum additional burden on the end user workflow.

To develop the CAT, we used the following process: first, biologists independently considered each of the terms available in the BAO and prioritized assignments for the CAT. Each assignment was associated with a number of possible values based on the BAO hierarchy. Then, quantitative and qualitative approaches were used to determine if the prioritized assignments included in the CAT were sufficient to fully describe most assays. For the quantitative approach, we assessed the set of 1066 PubChem bioassays ${ }^{27}$ that were previously annotated by hand by BAO experts. ${ }^{28}$ In that exercise, the BAO experts aimed to fully annotate each assay, capturing all applicable information for more than a hundred different categories or terms. If there was not an applicable value, the assignment or category was left blank. We analyzed the use of the BAO terms to assess the utility and comprehensiveness of the assignments included in the CAT compared to the remaining terms. We found that the 16 CAT assignments were annotated in $81 \%$ of the 1066 PubChem assays compared to $33 \%$ for the remaining terms. We also found that $95 \%$ of the values for CAT assignments were BAO terms rather than literal or non-URI based terms, compared to $63 \%$ in the remaining categories. These results suggested that the CAT includes assignments that are both relevant to the majority of assays as represented in PubChem and well covered by the BAO.

For an in-depth qualitative assessment of the CAT, biologists annotated a wide variety of assays, encompassing different assay types (e.g., cell viability, enzyme activity, binding, and ADMET), assay formats (e.g., cell-based, biochemical, microsome, organism, tissue, etc.), and assay design methods (e.g., ATP quantitation, cell number, immunoassays, gene expression, radioligand binding, etc), as summarized in Table 1 . We found that in many cases, both from assay descriptions available from PubChem and from in-house screening assay descriptions, the CAT captured much of the relevant information. For example, annotating an assay for cell viability (PubChem ID 427) shows that all but two of the 16 CAT assignments are readily 
319

320

321

322

323

324

325

326

327

328

329

330

331

332

333

334

335

336

337

338

339

340

341

342

343

344

345

346

347

348

349

350

351

352

353

354

355

356

357

358

359

360

361

362

363

364

365

annotated from the short descriptive information provided (Figure 8). 'Target' is left blank, as it is not applicable (this assay aims solely to identify cytotoxic compounds); 'Detection Instrument' was not noted. Similarly, as shown in Figure 9, all applicable CAT assignments (15 of the 16) are annotated from the description of a competitive binding assay (PubChem ID 440). Figure 9 also illustrates that multiple values can be annotated for a single assignment, enabling content from complex assays to be captured. Together, these two examples highlight that both cellbased and biochemical assays can be extremely well-suited to be annotated using the CAT.

However, there were some cases where the CAT was less effective in capturing important information. For example, 14 of the 16 CAT assignments could be annotated for PubChem ID 488847 , some with multiple values; however, the 'big picture' view of this rather complex primary assay is not as readily apparent from its 'CAT profile' as from a single sentence in the description (Figure 10). In addition, this PubChem record had extensive technical details such as reagent components, liquid handling volumes and instruments, times of incubation and plate processing steps, which could be important for identifying matching assays or interpreting the results. Another example of a poor fit for the CAT, as noted earlier, are in vivo assays. These are largely beyond the scope of this effort, which is currently constrained to terms defined by the BAO: key parameters such as route of administration, dose, dose units, type of model (e.g. xenograft, disease) are not well represented. These and other limitations will be addressed in the future by adding or extending the underlying ontologies.

Finally, as noted earlier, we designed the CAT to be a 'one-size-fits-most' template. A summary of assignments for the complete set of assays annotated in the course of developing the CAT shows we have achieved this (Table 1). One consequence of this 'one-size-fits-most' strategy is that certain attributes (such as those highlighted in green or red in Figures 8 and 9) have been omitted. Depending on one's perspective, these types of data (such as positive and negative controls, data processing/normalization steps, relevant disease indication, and specific protocol details such as pre-incubation of compounds with the target, time or temperature of an assay) could be viewed as essential. We decided to exclude this type of information from the CAT because of irregularity of appearance in bioassay descriptions, the lack of coverage by the $\mathrm{BAO}$, or incompatibility with the current data model. Expanding into this area is an opportunity for future development, and it should be noted that the CAT may be used as a starting point for templates that provide a set of assignment options that are customized for subcategories of assays, or even specific projects. We believe the next immediate step should be to apply our CAT to a large $(>10,000)$ set of assays, both to facilitate new meta-analyses and to identify potential gaps in annotation revealed by such studies.

\section{PubChem}

Possibly the most voluminous source of openly accessible bioassay data can be found on PubChem, which hosts more than 1.1 million assay records at the time of publication, and is growing rapidly. These are individually associated with the chemical structures of the compounds for which the measurements were made. Each of the assays is decorated with several descriptive fields that are essentially plain text, and which are populated by contributors during the upload process, or in some cases by an import script transferring data from other sources. While many of the entries contain a significant amount of detail, the phrasing style and level of detail varies considerably, often erring on the side of too little or too much information about the assay protocol.

Nonetheless, the PubChem assay collection represents one of the best and most convenient sources of data for annotation purposes, and for this reason we have added a feature to the BioAssay Template editor that explicitly searches for PubChem records, as shown in Figure 11. 
366

367

368

369

370

371

372

373

374

375

376

377

378

379

380

381

382

383

384

385

386

387

388

389

390

391

392

393

394

395

396

397

398

399

400

401

402

403

The dialog box allows the user to type in a PubChem Assay ID number, or to hit the button labelled Random, which picks an arbitrary assay from the entire collection, and fills in the corresponding text and URI of origin. While a large proportion of assays loaded into PubChem contain only sparse tags about the data source, or the abstract of the corresponding publication, there are a significant number of records that contain lengthy descriptions of the assay. The dialog box provides an opportunity for the user to tidy up the text (e.g. removing irrelevant content) prior to importing it into the schema. The content is then added to the list of assays being annotated within the schema model, whereby the origin is recorded as a link to the assay, and the text is associated using the hasParagraph predicate. Once the text is augmented with annotations using the current template, it becomes a useful entry for training data. This is one of our main strategies for generating a corpus of data for machine-learning purposes, which will ultimately find its way into a user friendly ELN for bioassay annotation.

\section{Analysis}

Because the data model we describe is based on semantic web triples, and the file format that is used by the BioAssay Schema Editor is made up of triples (in Turtle format), it means that any templates and assay annotations can be loaded directly into a triple store database, and queried using SPARQL queries. Content can be hosted on private servers for local use, or it can be exposed to the greater web of connected data. The supplementary information (Section 1) describes a configuration script for the open source Apache Fuseki Jena server which can be used to load the BioAssay Ontology, its related ontologies, and some number of files saved with the BioAssay Schema Editor, which can then be served up as read-only content.

Once the content is available via a SPARQL endpoint, there are a number of boilerplate queries that can be used to extract summary and specific information. Fetching a list of all bioassay templates can be accomplished using the following query:

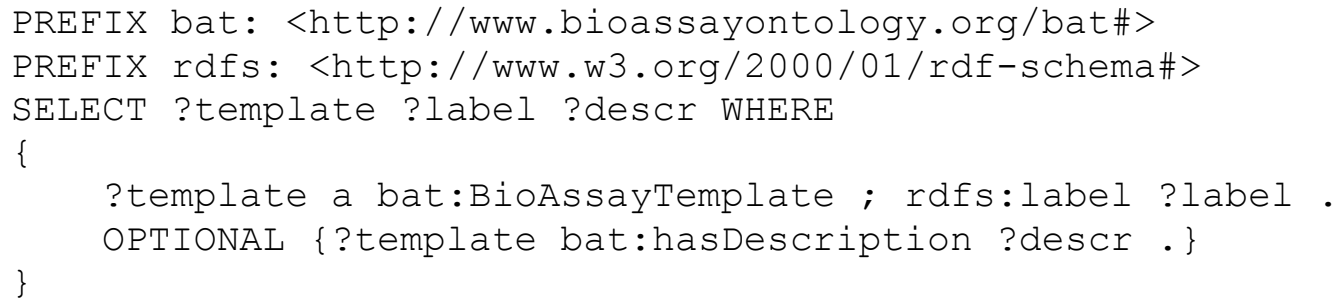

The above query identifies any resource that is tagged as having the BioAssayTemplate type. Obtaining information about the assignments that are associated with a template can be done by looking for resources of type Group that are associated with it. Obtaining a summary list of assignments that are attached to the top level (i.e. not within a subgroup) can be accomplished with a query similar to the following (using the same prefixes as above) which explicitly references the common assay template: 


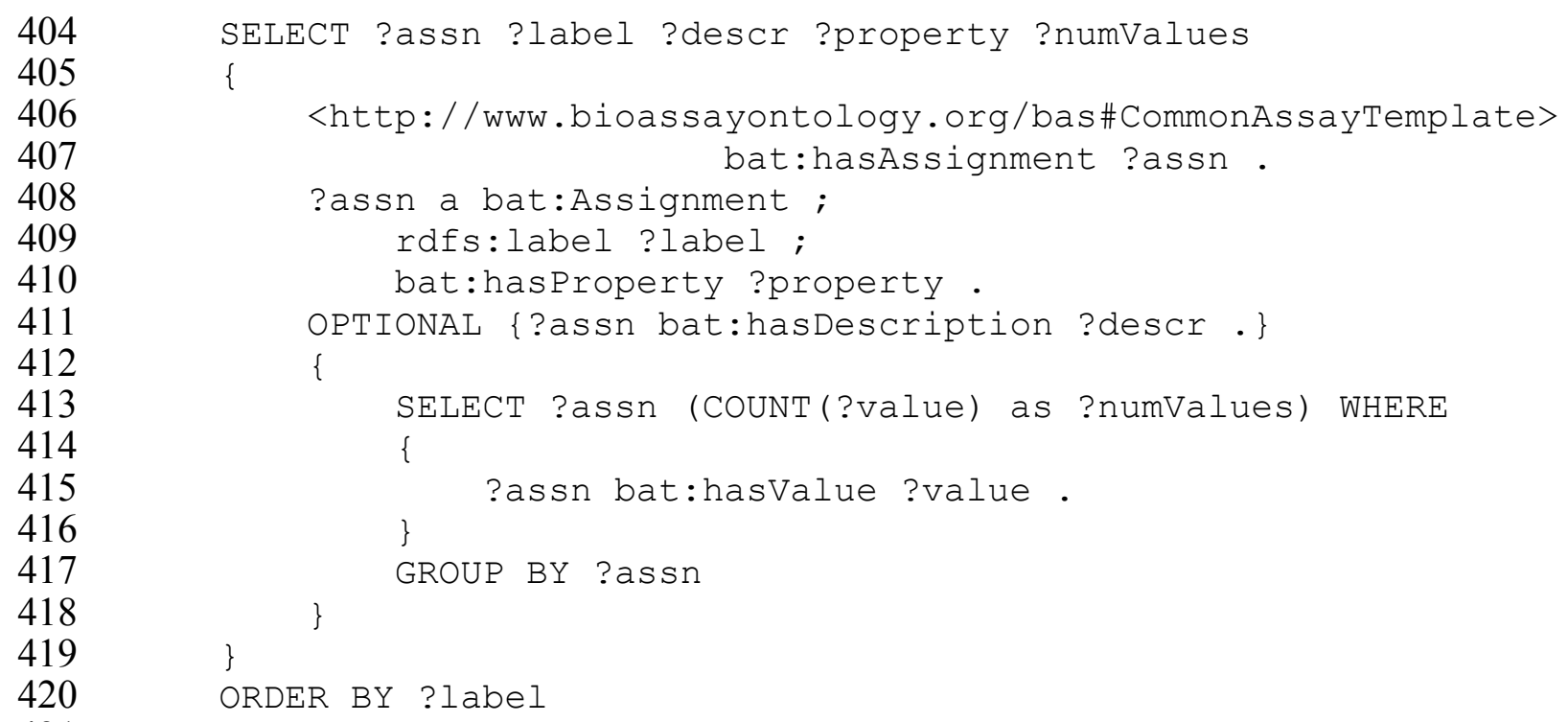

404

405

406

407

408

409

410

411

412

413

414

415

416

417

418

419

420

421

422

423

424

425

426

427

428

429

430

431

432

433

434

435

436

437

438

439

440

441

442

443

444

445

Similarly, assignments with one level of nesting can be obtained with a slightly longer query, which explicitly inserts a subgroup in between the template and assignment:

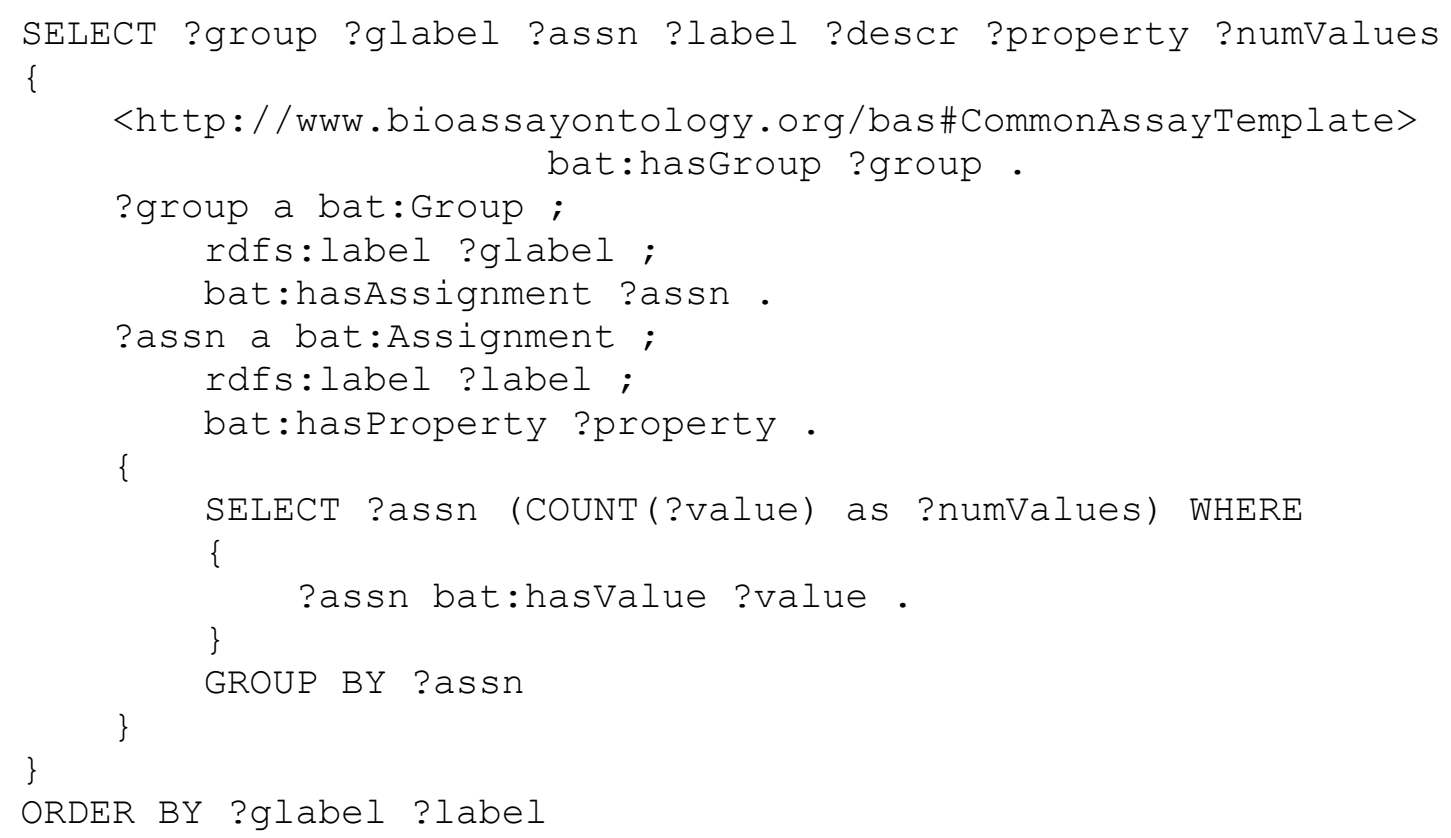

To query for information about the prescribed values for assignment (in this case the bioassay assignment from the common assay template), the following query can be used: 
446

447

448

449

450

451

452

453

454

455

456

457

458

459

460

461

462

463

464

465

466

467

468

469

470

471

472

473

474

475

476

477

478

479

480

481

482

483

484

485

486

487

488

489

490

491

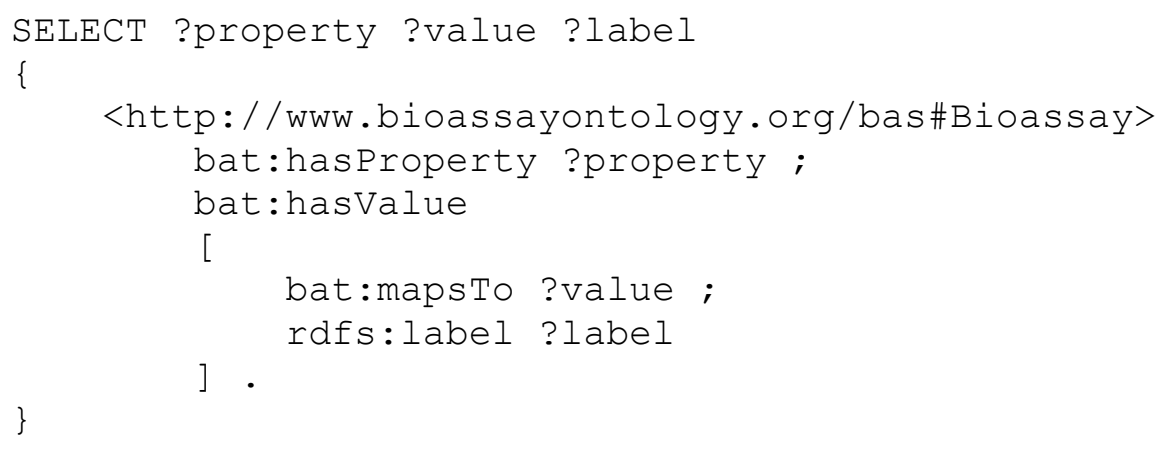

The query specifically pulls out the property field, which is typically a link into the BAO property terms, and the value field, which is typically a link into the BAO classes. Pursuing either of these resources provides a wealth of implicit information, partly from the hierarchical nature of the $\mathrm{BAO}$ terms, and the unlimited opportunities for these terms to be linked to other semantic resources.

To obtain a list of assays that have been annotated using one of the templates, the following query can be used:

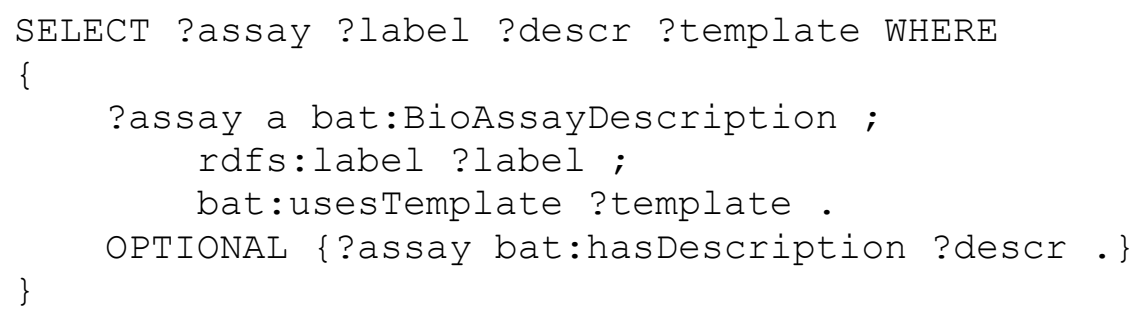

Obtaining all of the annotations for such an assay can be done with:

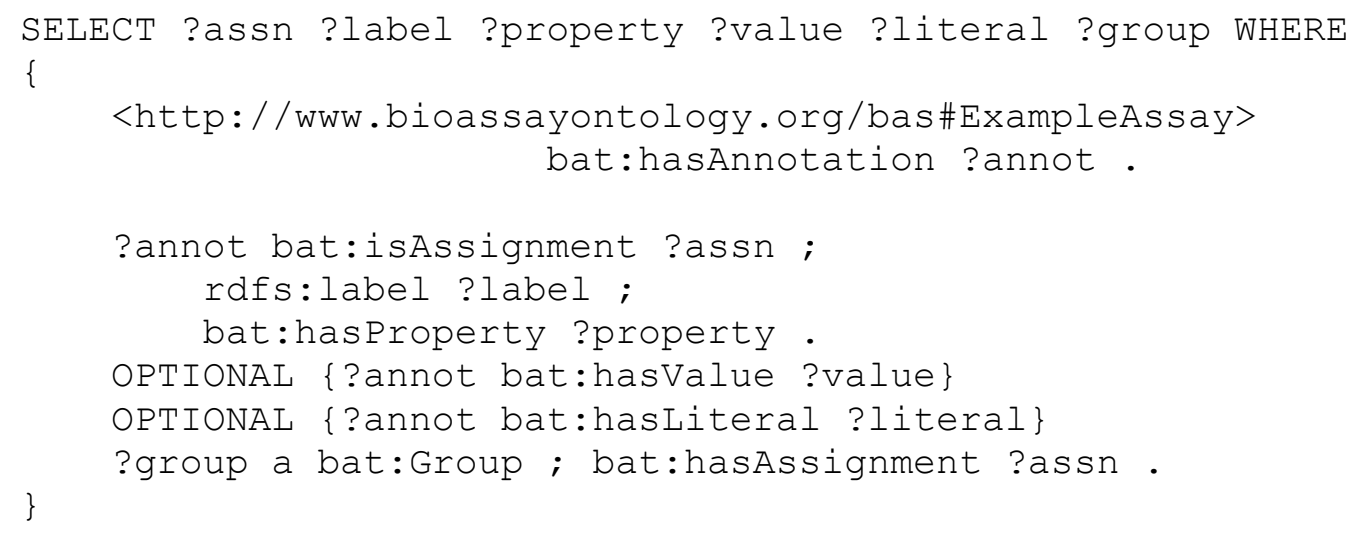

Because annotations are directly attached to an assay description, hierarchical information about the nature of the assignment can be obtained by further investigating the template definition of the assignment (?assn) or either of the linked BAO terms (?property and ?value).

\section{Conclusion}

We have developed a data model and interactive tool that can be used to narrow the degrees of freedom from the BioAssay Ontology (BAO) and its linked dependencies. This has been done in 
492 order to facilitate content creation activities, so that semantic annotation of assay protocols can

493 be carried out by a domain expert with no corresponding expertise with the underlying ontology.

494

495 We have provided a proof of concept tool that creates a user interface based on the template data model, and made this available to the community as open source.

496 The data model that we have created follows a simplistic pattern, where elementary facts can

497

498

499 be asserted. By leveraging the implied value of the underlying ontology, a small collection of a dozen or so such annotations provides a significant amount of machine-readable context about the assay. While insufficient to completely define an assay protocol experiment, this stands in contrast to the standard practice of providing essentially zero machine-readable information (i.e. plain English text with quasi-standardized jargon).

502

503

504

505

506

We have made available the common assay template (CAT) which was designed by biologists with the objective of leveraging the BAO to provide the largest amount of useful, relevant, machine-readable information with the fewest number of additional data points needing to be captured by the originating scientist. The CAT is expected to be useful for a wide variety of sorting, filtering, and data aggregating tasks that drug discovery scientists need to be able to carry out on a large scale, but currently cannot due to the absence of machine-readable annotations.

The CAT prioritizes 16 assignments that biologists consider most central to describing their

510 assays and reporting assay results. Annotations for these assignments will enable biologists to

511 ask complex queries. For example, one could ask if there are systematic differences in cell-

512 based versus biochemical-based assays for a certain target class, such as kinases. One could

513 determine if a certain assay set-up, such as 96-well plates using a spectrophotometer were

514 likely to have a higher hit rate. Similarly, one could identify if a certain compound or class of

515 compounds is active in multiple assays, and if those assays assess similar biological processes

516 or if the activity is likely to be an artifact.

517 By focusing on 16 assignments out of more than a hundred options available in the BAO, the

518 CAT is meant to impose a minimal burden for annotating scientists. Our goal is to make

519 annotating assays simple and easy so that the practice may be generally adopted. Templates

520 are malleable and scientists can easily include other assignments.

521 One critical type of information that is not included in the current framework is protocol steps, which would be essential for directly comparing two assays. In the future, it would be useful if this information were machine-readable. However, semantic technology using a simplistic data model like the BAT cannot capture sequences of information. Capturing procedural or protocol steps would require the development of a more complex data model. Under the current system, we imagine that queries using annotations from the CAT will allow scientists to hone in on similar assays, but for the moment, experts will still need to read the full assay descriptions to make decisions about combining different assays' data sets.

We have carried out this work in the context of a much larger scope, which is to provide scientists with tools to easily annotate bioassays and other related experiments in a way that is complete and machine-readable. Given that the standard industry practice does not involve adding any machine readable data to assay protocols, and that there are currently no widely available tools to do so with a user experience that is sufficiently painless for mass adoption, we have taken an incremental approach. This additional work has been done in order that we can continue with our previous work that was focused on using machine learning techniques to accelerate manual assignment of assays. ${ }^{15}$ Our immediate follow-up goals are to make use of the CAT to gather a large corpus of training data, both from active users of CDD Vault, and from existing repositories such as PubChem. This training data will be used to ensure that our 
539

540

541

542

543

544

545

546

547

548

549

550

551

552

553

554

555

556

557

558

559

560

561

562

563

564

565

566

567

568

569

570

571

572

573

574

575

enterprise ELN tools will be supported by machine learning technology as soon as they are unveiled.

We are also pursuing options for extending the BioAssay Template (BAT) data model so that it is capable of capturing more sophisticated information about assays, e.g. linking to other ontologies to cover more types of assays; adding terminology for capturing quantities; addition of indefinite numbers of preparation steps; dependent assignment types, etc. One critical step when we enable connecting with other ontologies will be the ability to link the 'Target' to a unique identifier such as geneid or UniProtID. Each unique target identifier can be associated with a rich array of corresponding GO terms, of which a subset are mapped into the default selection of BAO classes. This will enable comparison of assays based on specific targets and related biological processes or molecular functions. While our first objective is horizontal scaling, i.e. ensuring that all assay protocols have semantic annotations that make a large portion of the content machine-readable, pursuing vertical scaling is also of great interest, i.e. making it possible for the semantic annotations to replace the need for use of English text. ${ }^{29}$ This brings about some exciting possibilities beyond just improvement of searching and matching, such as uploading protocols to robotic assay machinery, or making the publication process multi-lingual, thus alleviating a considerable burden to non-native English speakers. Pursuing this goal will require significant additions to the BAO itself, as well as making increased use of borrowed terms from other ontologies.

The technology that we have described in this article has been created for the purpose of improving the electronic lab notebook (ELN) technology that is offered by Collaborative Drug Discovery, Inc. (CDD), and we have begun work on a web-based interface for using templates such as the CAT for annotating assay protocols. ${ }^{30}$ We have disclosed all of the underlying methods, data and open source code because we welcome participation by anyone and everyone. While CDD is a privately held for-profit company, it is our firm belief that improvement to this particular aspect of scientific research is a positive sum game, and we have more to gain by sharing than by keeping our technology entirely proprietary.

\section{Supporting Materials}

The BioAssay Schema Editor is publicly available from GitHub (https://github.com/cdd/bioassaytemplate). The source code for the application is available under the terms of the Gnu Public License (GPL) v2, which requires that derived works must also be similarly open. The underlying semantic data model for the template and assay annotation, as well as the common assay template (CAT), are public domain: they are not copyrighted, and no restrictions are placed on their use. The BioAssay Ontology (BAO) is available from the corresponding site (http://bioassayontology.org/bioassayontology) under the Creative Commons Attribution License v3. 
576 Tables

Table 1. Representation of Common Assay Template in Sample Assay Set

\begin{tabular}{|l|c|c|}
\hline CAT Assignment & $\begin{array}{c}\text { Test Assays (of 43) } \\
\text { With at Least 1 Value }\end{array}$ & $\begin{array}{c}\text { \# of Unique } \\
\text { Values Annotated }\end{array}$ \\
\hline bioassay type & $43(100 \%)$ & 24 of 88 \\
\hline assay format & $43(100 \%)$ & 6 of 19 \\
\hline assay design method & $43(100 \%)$ & 20 of 76 \\
\hline assay cell line & $24(55.8 \%)$ & 15 of 95 \\
\hline organism & $41(95.3 \%)$ & 11 of 65 \\
\hline biological process & $40(93.0 \%)$ & 28 of 54 \\
\hline target & $32(74.4 \%)$ & 13 of 38 \\
\hline assay mode of action & $43(100 \%)$ & 8 of 13 \\
\hline result & $41(100 \%)$ & 16 of 94 \\
\hline result unit of measurement & $32(74.4 \%)$ & 6 of 56 \\
\hline assay screening campaign stage & $40(93.0 \%)$ & 8 of 23 \\
\hline assay footprint & $36(83.7 \%)$ & 5 of 20 \\
\hline assay kit & $9(20.9 \%)$ & 5 of 93 \\
\hline physical detection method & $42(97.7 \%)$ & 11 of 51 \\
\hline detection instrument & $26(60.5 \%)$ & 9 of 97 \\
\hline perturbagen type & $20(46.5 \%)$ & 3 of 9 \\
\hline
\end{tabular}

Figure Captions

579 Figure 1: A selection of the BioAssay Ontology hierarchy, visualized using BioPortal

580 (http://bioportal.bioontology.org): (a) classes and (b) properties.

581 Figure 2: An overview of the common assay template (CAT) at the time of publication.

582 Figure 3: BioAssay Template data model, which is used to describe a template.

583 Figure 4: Data model for annotated assays, which is used to apply a template to a specific 584 assay.

585 Figure 5: A snapshot of the BioAssay Schema Editor. On the left hand side the current template 586 is shown at the top (with its hierarchy of groups and assignments), and any assays currently in 587 progress shown underneath. The panel on the right shows the details for an assignment - assay 588 format - and the prescribed values that are associated with it.

589 Figure 6: A snapshot of the two main tabs used for locating a value in the BioAssay Ontology. 590 The left hand side (a) shows the list view, which is flat, while the right hand side (b) shows the 591 values in context of the actual hierarchy of the underlying ontology. 
592 Figure 7: A snapshot of the annotation interface that is available within the template editor (a).

593 The current template can be applied to specific assays within the same overall user interface,

594 which is a convenient way to evaluate its suitability. Selecting any of the assignments brings up

595 a dialog box presenting all of the prescribed values (b).

596 Figure 8. Example of PubChem Assay text ideally suited for annotation with the CAT.

597 Left: Text from description in PubChem Assay ID 427: yellow = information captured in CAT,

598 green = information not captured but possible for a future version (e.g., controls, data

599 processing), red= information beyond the scope of BAO (technical details) Right: CAT

600 assignments in BioAssay Schema Editor.

601 Figure 9. Example of PubChem Assay text ideally suited for annotation with the CAT.

602 Left: Text from description in PubChem Assay ID 440: yellow = information captured in CAT,

603 pink = information added as 'literal' values (i.e., too specific to exist as a BAO entry, but deemed

604 valuable), green = information not captured but possible for a future version (e.g., controls, data

605 processing), red= information beyond the scope of BAO (technical details). Right: CAT

606 assignments in BioAssay Schema Editor. Annotations added as 'literal' values are highlighted

607 yellow and contained in single quotes. Note that multiple values for a single CAT assignment

608 can be annotated (target biological process, assay mode of action, assay screening campaign

609 stage, perturbagen type).

610 Figure 10. Example of an assay partially suited for annotation with the CAT. Left: Text

611 from description in PubChem Assay ID 488847: yellow = information captured in CAT, pink=

612 information added as 'literal' values (i.e., too specific to exist as a BAO entry, but deemed

613 valuable), green = information not captured but possible for a future version (e.g., controls,

614 labels of target and ligand, assay quality data (Z')), red= information beyond the scope of BAO

615 (technical details). Right: CATvalues assigned in the BioAssay Schema Editor capture key

616 parameters of the assay yet do not capture the complexity of the assay articulated in the single

617 sentence (arrow): "a flow cytometry protein interaction assay to screen for compounds that

618 compete with RNA binding to GRK2".

619 Figure 11: Dialog box for random lookup of assays from PubChem.

\section{References}

1. A.M. Clark, A.J. Williams, S. Ekins: Machines first, humans second: on the importance of algorithmic interpretation of open chemistry data, Journal of Cheminformatics, 7:9 (2015) DOI 10.1186/s13321-015-0057-7

2. A. Hersey, S. Senger, J.P. Overington: Open data for drug discovery: learning from the biological community. Future Medicinal Chemistry, 4, 1865-1867 (2012) DOI

10.4155/fmc. 12.159

3. G.F. Ecker, B. Williams-Jones: Editorial: Open Innovation in Drug Discovery. Molecular Informatics, 31, 519-520 (2012) DOI 10.1002/minf.201280004

4. A.J. Williams, J. Wilbanks, S. Ekins S: Why Open Drug Discovery Needs Four Simple Rules for Licensing Data and Models. PLoS Computational Biology, 8:e1002706 (2012) DOI 10.1371/journal.pcbi.1002706

5. K.Y. Helal, M. Maciejewski, E. Gregori-Puigjane, M. Glick, A.M. Wassermann: Public Domain HTS Fingerprints: Design and Evaluation of Compound Bioactivity Profiles from 
PubChem's Bioassay Repository. Journal of Chemical Information and Modeling, just accepted (2016) DOI 10.1021/acs.jcim.5b00498

6. E. Bolton: Reporting biological assay screening results for maximum impact, Drug Discovery Today: Technologies, 14, 31-36 (2015) DOI 10.1016/j.ddtec.2015.03.004

7. It should be noted that the majority of the first million PubChem assays do not contain detailed experimental assay descriptions. Contributors such as the Broad Institute and organizations affiliated with the Molecular Libraries Screening Center can be selected by browsing the sources: http://pubchem.ncbi.nlm.nih.gov/sources/sources.cgi

8. A. Gaulton, L.J. Bellis, A.P. Bento, J. Chambers, M. Davies, A. Hersey, Y. Light, S. McGlinchey, D. Michalovich, B. Al-Lazikani, J.P. Overington: ChEMBL: a large-scale bioactivity database for drug discovery. Nucleic Acids Research, D1100-D1107 (2012) DOI 10.1093/nar/gkr777

9. The materials for the BioAssay Ontology can be found at http://bioassayontology.org 10. S. Abeyruwan, U.D. Vempati, H. Küçük, U. Visser, A. Koleti, A. Mir, K. Sakurai, C. Chung, J. Bittker, P. Clemons, S. Brudz, A. Siripala, A. Morales, M. Romacker, D. Twomey, S. Bureeva, V. Lemmon, S.C. Schürer: Evolving BioAssay Ontology (BAO): Modularization, Integration and Applications, Journal of Biomedical Semantics, 5, 1:S5 (2014) DOI 10.1186/2041-1480-5-S1-S5 11. U.D. Vempati, M.J. Przydzial, C. Chung, S. Abeyruwan, A. Mir, K. Sakurai, U. Visser, V.P. Lemmon, S.C. Schürer: Formalization, annotation and analysis of diverse drug and probe screening assay datasets using the BioAssay Ontology (BAO). PLoS One, e49198 (2012) DOI: 10:1371/journal.pone.0049198

12. S. Kim, P.A. Thiessen, E.E. Bolton, J. Chen, G. Fu, A. Gindulyte, L. Han, J. He, S. He, B.A. Shoemaker, J. Wang, B. Yu, J. Zhang, S.H. Bryant: PubChem Substance and Compound databases. Nucleic Acids Research, D1202-13 (2016) DOI: 10.1093/nar/gkv951

13. E.L. Willighagen, A. Waagmeester, O. Spjuth, P. Ansell, A.J. Williams, V. Tkachenko, J. Hastings, B. Chen, D.J. Wild: The ChEMBL database as linked open data. Journal of Cheminformatics, 5:23 (2013) DOI: 10.1186/1758-2946-5-23

14. A. de Souza, J.A. Bittker, D.L. Lahr, S. Brudz, S. Chatwin, T.I. Oprea, A. Waller, J.J. Yang, N. Southall, R. Guha, S.C. Schürer, U.D. Vempati, M.R. Southern, E.S. Dawson, P.A. Clemons, T.D.Y. Chung: An Overview of the Challenges in Designing, Integrating, and Delivering BARD: A Public Chemical-Biology Resource and Query Portal for Multiple Organizations, Locations, and Disciplines. Journal of Biomedical Screening, 19, 614-627 (2014) DOI: $10.1177 / 1087057113517139$

15. A.J. Williams, L. Harland, P. Groth, S. Pettifer, C. Chichester, E.L. Willighagen, C.T. Evelo, 7, N. Blomberg, G. Ecker, C. Goble, B. Mons: Open PHACTS: semantic interoperability for drug discovery. Drug Discovery Today, 17, 1188-1198 (2012) DOI:

10.1016/j.drudis.2012.05.016

16. The Gene Ontology Consortium. Gene Ontology Consortium: going forward. (2015) Nucl Acids Res 43 Database issue D1049-D1056 Online at Nucleic Acids Research

17. It can also be browsed and edited using software such as Protégé, which can be found at http://protege.stanford.edu

18. A.M. Clark, B.A. Bunin, N.K. Litterman, S.C. Schürer, U. Visser: Fast and accurate semantic annotation of bioassays exploiting a hybrid of machine learning and user confirmation. PeerJ, 2:e524 (2014) DOI 10.7717/peerj.524

19. A. de Souza, J.A. Bittker, D.L. Lahr, S. Brudz, S. Chatwin, T.I. Oprea, A. Waller, J.J. Yang, N. Southall, R. Guha, S.C. Schürer, U.D. Vempati, M.R. Southern, E.S. Dawson, P.A. Clemons, T.D.Y. Chung: An Overview of the Challenges in Designing, Integrating, and 
Delivering BARD: A Public Chemical-Biology Resource and Query Portal for Multiple Organizations, Locations, and Disciplines. Journal of Biomedical Screening, 19, 614-627 (2014) DOI: $10.1177 / 1087057113517139$

20. Y. Wang Y, T. Suzek, J. Zhang, J. Wang, S. He, T. Cheng, B.A. Shoemaker, A. Gindulyte, S.H. Bryant: PubChem BioAssay: 2014 update. Nucleic Acids Research, D1075-82 (2014) DOI 10.1093/nar/gkt978

21. S.C. Schürer, U. Vempati, R. Smith, M. Southern, V. Lemmon: BioAssay ontology annotations facilitate cross-analysis of diverse high-throughput screening data sets. Journal of Biomolecular Screening, 16:415-426 (2011) DOI 10.1177/1087057111400191

22. See W3C Resource Description Framework: http://www.w3.org/RDF

23. See W3C RDF Turtle: http://www.w3.org/TR/turtle

24. Gnu Public License 2.0: http://www.gnu.org/licenses/gpl-2.0.en.html: the license allows anyone to use the source code for any purpose, on the condition that products making use of it must be made available under a license that is at least as open. Copyright for the project is held by Collaborative Drug Discovery, Inc.

25. See Apache Jena project: http://jena.apache.org

26. Downloadable OWL files for the BioAssay Ontology:

http://bioassayontology.org/bioassayontology

27. Y. Wang Y, T. Suzek, J. Zhang, J. Wang, S. He, T. Cheng, B.A. Shoemaker, A. Gindulyte, S.H. Bryant: PubChem BioAssay: 2014 update. Nucleic Acids Research, D1075-82 (2014) DOI 10.1093/nar/gkt978

28. S.C. Schurer, U. Vempati, R. Smith, M. Southern, V. Lemmon: BioAssay ontology annotations facilitate cross-analysis of diverse high-throughput screening data sets. Journal of Biomolecular Screening, 16:415-426 (2011) DOI 10.1177/1087057111400191

29. L.N. Soldatova, D. Nadis, R.D. King, P.S. Basu, E. Haddi, V. Baumié, N.J. Saunders, W. Marwan, B.B. Rudkin: EXACT2: the semantics of biomedical protocols. BMC Bioinformatics, 15, S5 (2014) DOI: 10.1186/1471-2105-15-S14-S5

30. A preliminary version of the web interface can be found at http://bioassayexpress.com. At the time of writing this service is in an early pre-alpha phase, but will be updated as the project progresses. 


\section{1}

A selection of the BioAssay Ontology hierarchy, visualized using BioPortal (http://bioportal.bioontology.org): (a) classes and (b) properties.

Figure 1: A selection of the BioAssay Ontology hierarchy, visualized using BioPortal (http://bioportal.bioontology.org): (a) classes and (b) properties.

(a)

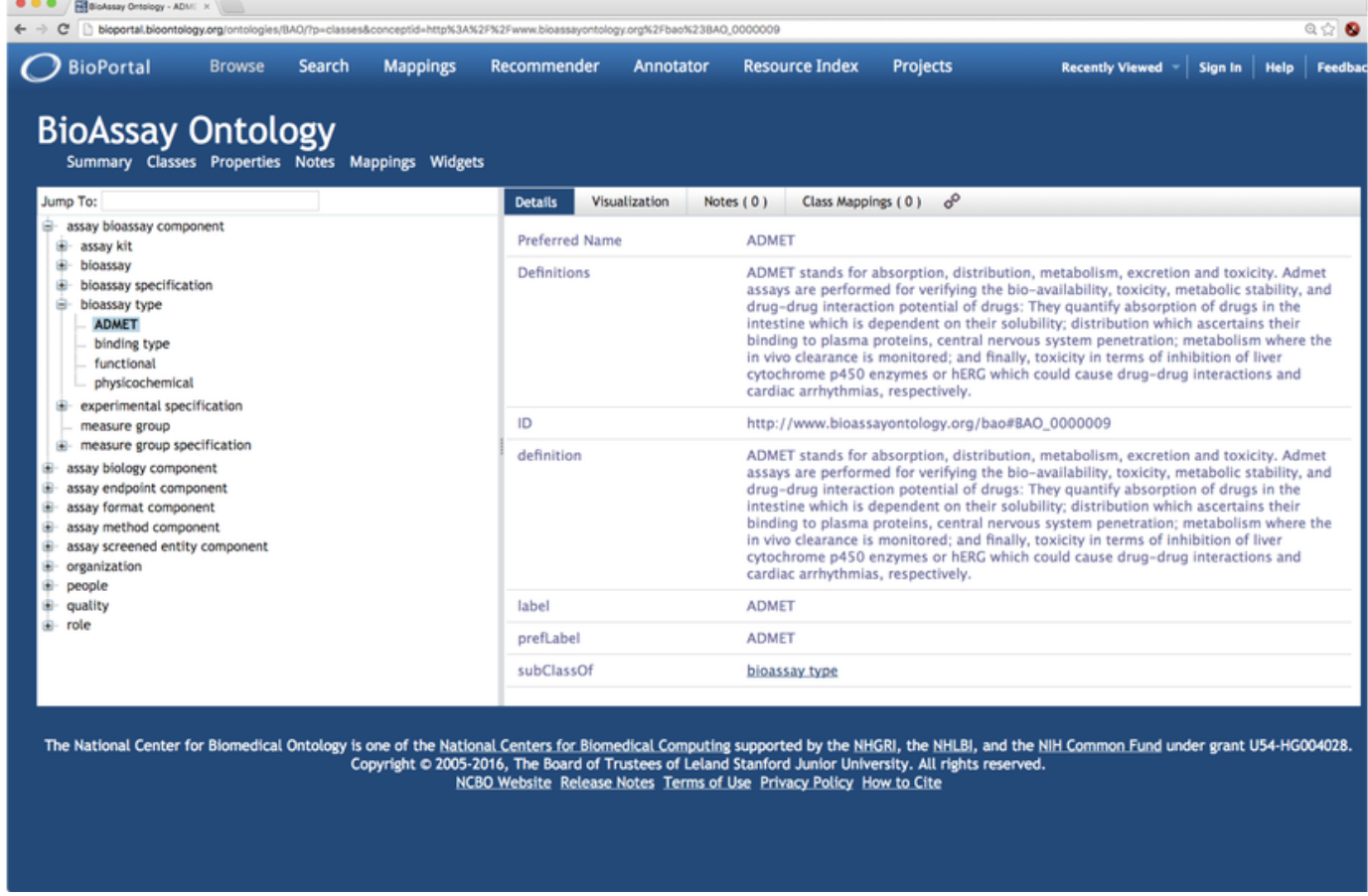

(b)

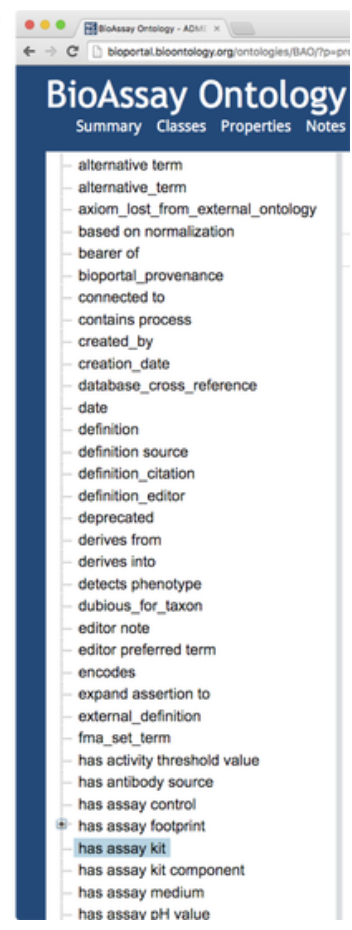


Figure 2 (on next page)

An overview of the common assay template (CAT)

Figure 2: An overview of the common assay template (CAT) at the time of publication. 


\section{bioassay type $\longrightarrow$ ADMET}

has bioassay apoptosis assay

be galactosidase enzyme activy assay

beta galactosidase reporter gene assay

beta lactamase reporter gene assay

binding assay

bioavailability assay

calcium redistribution assay

cAMP redistribution assay

(+ 79 more)

assay format $\longrightarrow$ biochemical format

assay format $\longrightarrow$ cell based format

cell membrane format

cell-free format

cytosol format

microsome format

mitochondrion format

nuclear extract format

nucleic acid format

(+ 10 more)

\section{assay design method $\longrightarrow$ antigen down assa}

has assay design method

ATP quantitation

ATP quantitation using luciferase

beta galactosidase induction

beta lactamase induction

binding assessment method

caspase activity determination

cell cycle progression assessment method

cell movement measurement method

(+ 67 more)

assay cell line $\longrightarrow 293 \mathrm{ce}$

$\underset{\text { is cell line of }}{\text { assan cell line } \longrightarrow \text { 293T/17 cell }}$

A2780

A549 cell

ACHN cell

AML12 cell

BA/F3 cell

BJ

BSC-1 86 more

$$
\text { (+ } 86 \text { more) }
$$

organism $\longrightarrow$ Arabidopsis thaliana has organism

\section{bacterium}

Bluetongue virus 10

Bos taurus

Caenorhabditis elegans

Candida albicans

Canis lupus familiaris

cellular organisms

Chlorocebus aethiops

(+ 56 more)

biological process $\longrightarrow$ absenc

biological proce?

alternative mRNA splicing, via spliceosome

ambiguous

apoptotic process

autophagy

biofilm formation

calcium-mediated signaling using intracellular calcium source_bao

target has biological macromolecule

adhesion

carbohydrate

chaperone

cytosolic protein

enzyme

enzyme regulator

G protein

$G$ protein coupled recepto

generic hydrolase

(+ 29 more)

assay mode of action $\longrightarrow$ activation

has mode of action agonism

antagonism

inhibition

irreversible binding

ligand binding mode of action

ligand function mode of action

modulation

result $\longrightarrow 50$ percent activation

has result 50 percent inhibition

80 percent inhibition

90 percent inhibition

AC10 absolute

AC1000 absolute

AC26 absolute

AC35 absolute

AC40 absolute
(+ 85 more)

result unit of measurement $\longrightarrow$ angstrom

catalytic (activity) concentration unit cell concentration unit

cells per milliliter

centimeter

century

concentration unit

concentration unit

counts per second

(+ 47 more)

assay screening campaign stage $\longrightarrow$ alternate assay condition has assay stage alternate assay format alternate assay type alternate cell line assay alternate confirmatory assay alternate organism assay alternate target assay compound aggregation assay compound fluorescence assay (+ 14 more)

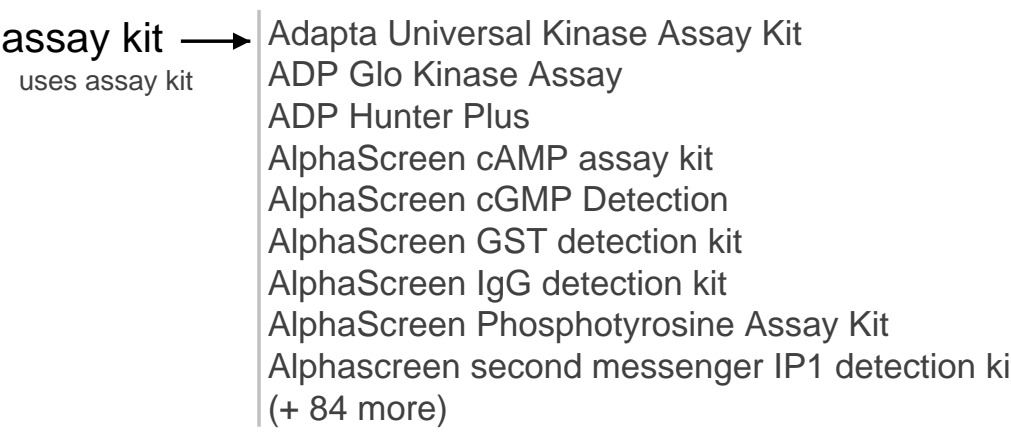

assay kit $\longrightarrow$ Adapta Universal Kinase Assay Kit uses assay kit ADP Glo Kinase Assay

ADP Hunter Plus

AlphaScreen cAMP assay kit

Alphascreen cGMP Detection

Alphascreen GST detection kit

AlphaScreen IgG detection kit

AlphaScreen Phosphotyrosine Assay Kit

Alphascreen second messenger IP1 detection $\mathrm{k}$

Alphascreen
(+ 84 more $)$

physical detection method $\longrightarrow$ absorbance has detection method

alphascreen

atomic absorption spectrophotometry

bio layer interferometry

bioluminescence

brightfield microscopy

carbon nanotube based sensor

chemiluminescence

circular dichroism

(+ 42 more)

detection instrument $\longrightarrow$ 3i Mariana

\begin{tabular}{l|l} 
uses detection instrument & $8453 \mathrm{UV}$-Visible Spectrophotometer
\end{tabular}

Acumen

AlphaQuest reader

AMINCO-Bowman Series 2 Luminescence Spectrometer Analyst $\mathrm{HT}$

API 4000 LC/MS/MS System

Applied biosystems 8200

ArrayScan 3.1 HCS Reader

(+ 88 more)

perturbagen type $\longrightarrow$

$\begin{array}{lll}\text { has perturbagen } & \text { DIVERSet }\end{array}$

LOPAC 1280

miRNA library

MLSMR library

NINDS library

shRNA library

siRNA library

The NatProd Collection

CAM-mediated signaling $B A O$

cell cycle

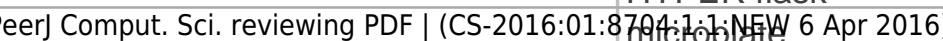

536 well plate

24 well plate

96 well plate

array

gene array

+ 45 more)

(+ 11 more) 
Figure 3 (on next page)

BioAssay Template data model

Figure 3: BioAssay Template data model, which is used to describe a template. 


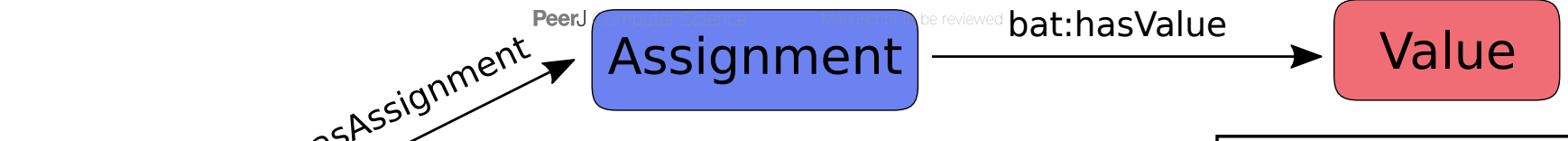

rdf:type bat:BioAssayTemplate rdf:type bat:Group rdfs:label ^^xsd:string bat:hasDescription $\wedge \wedge x$ xd:string

rdf:type bat:Assignment

rdfs:label $\wedge \wedge x$ xd:string

bat:hasDescription $\wedge^{\wedge} x$ xd:string

bat:hasProperty reference rdfs:label $\wedge \wedge x s d: s t r i n g$ bat:hasDescription $\wedge^{\wedge} x$ sd:string bat:mapsTo reference

\section{Assignment}


Figure 4 (on next page)

Data model for annotated assays

Figure 4: Data model for annotated assays, which is used to apply a template to a specific assay. 
rdf:type bat:BioAssayDescription rdfs:label ^^xsd:string

bat:hasDescription $\wedge \wedge x s d$ :string bat:usesTemplate reference bat:hasParagraph ^^xsd:string bat:hasOrigin reference

Annotation

isAssignment reference rdfs:label ^^xsd:string bat:hasDescription ^^^xsd:string bat:hasProperty reference bat:hasValue reference bat:hasLiteral Iiteral 
5

A snapshot of the BioAssay Schema Editor

Figure 5: A snapshot of the BioAssay Schema Editor. On the left hand side the current template is shown at the top (with its hierarchy of groups and assignments), and any assays currently in progress shown underneath. The panel on the right shows the details for an assignment - assay format - and the prescribed values that are associated with it.

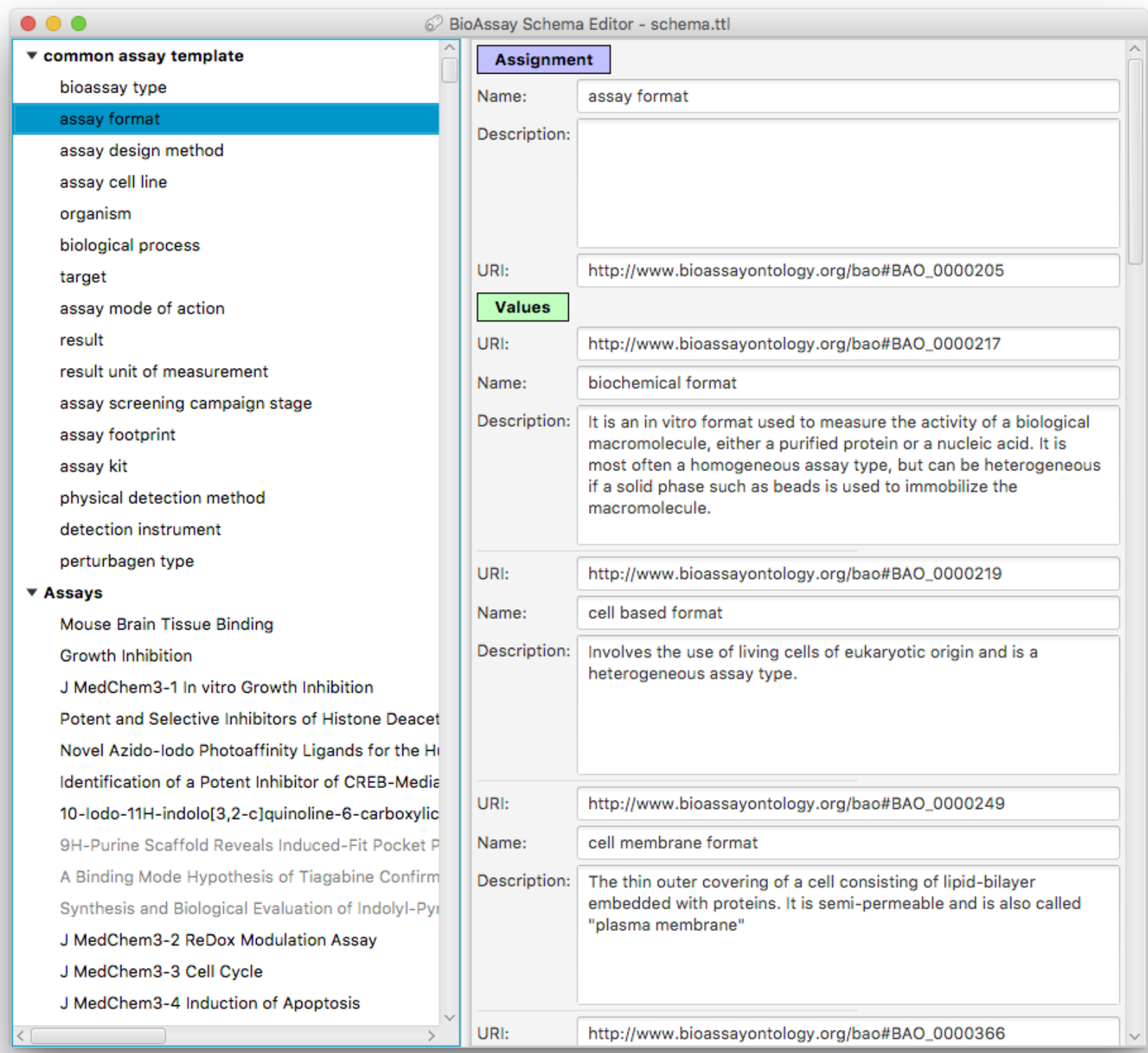


6

A snapshot of the two main tabs used for locating a value in the BioAssay Ontology

Figure 6: A snapshot of the two main tabs used for locating a value in the BioAssay

Ontology. The left hand side (a) shows the list view, which is flat, while the right hand side (b) shows the values in context of the actual hierarchy of the underlying ontology.

(a)

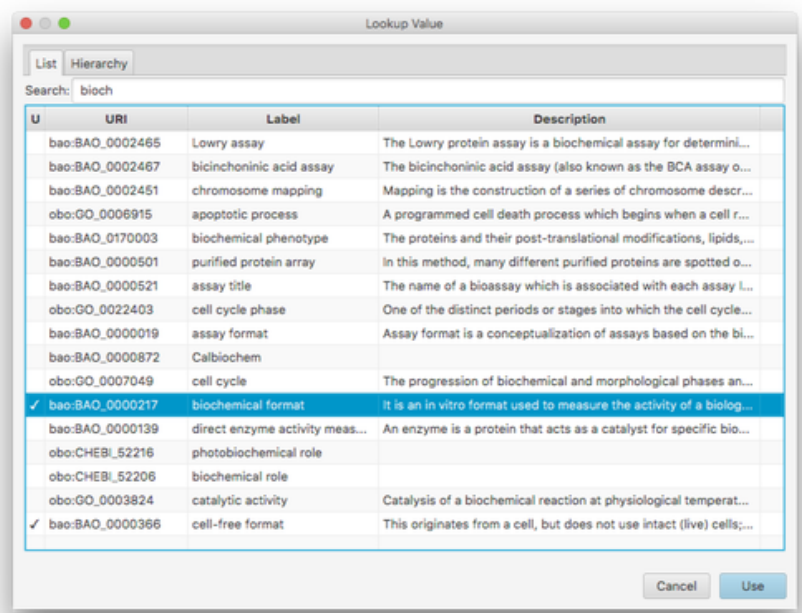

(b)

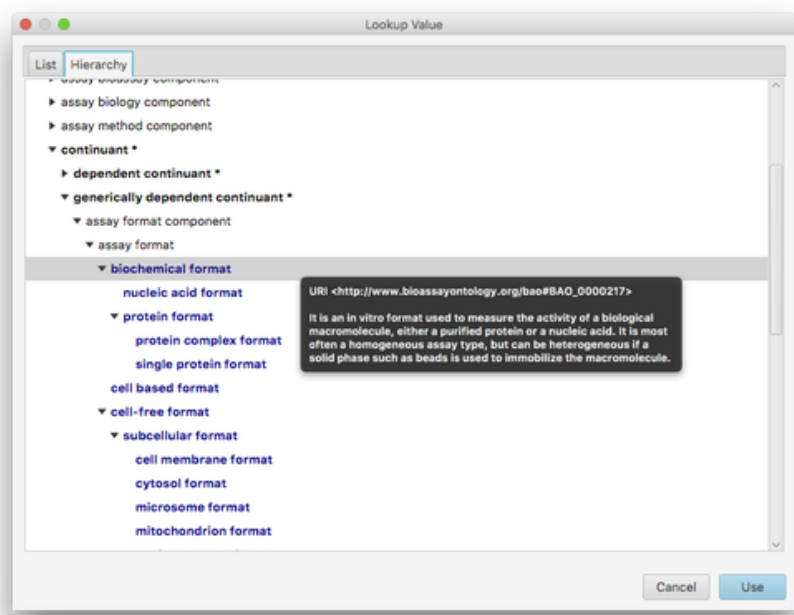


7

A snapshot of the annotation interface that is available within the template editor

Figure 7: A snapshot of the annotation interface that is available within the template editor (a). The current template can be applied to specific assays within the same overall user interface, which is a convenient way to evaluate its suitability. Selecting any of the assignments brings up a dialog box presenting all of the prescribed values (b).

(a)

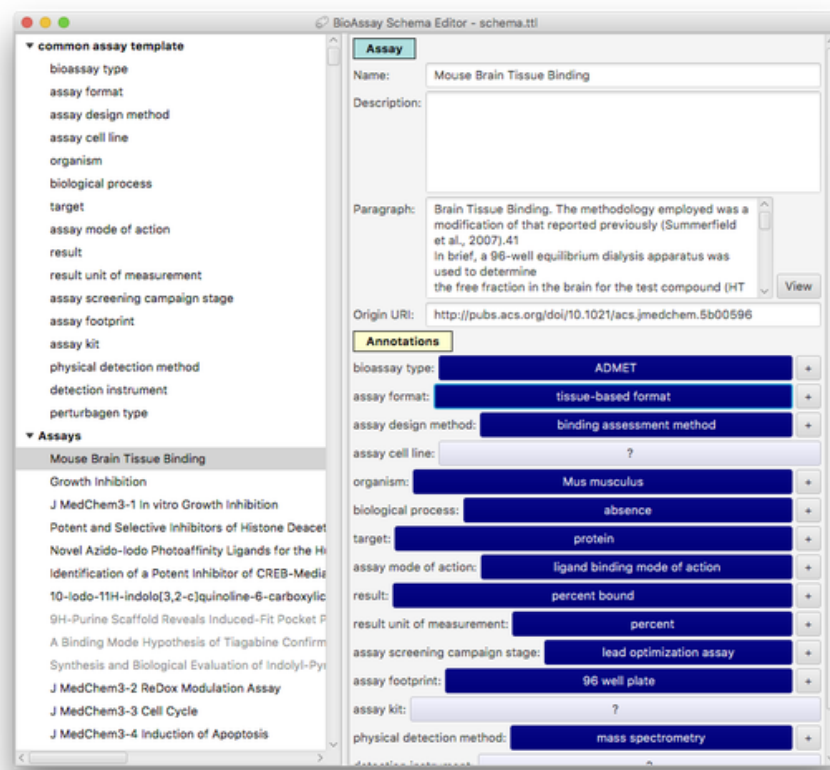

(b)

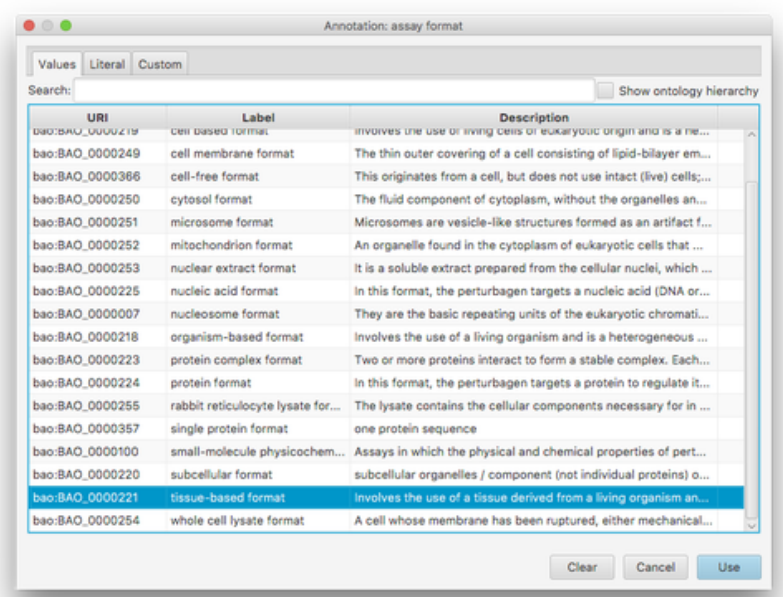


Figure 8 (on next page)

First example of PubChem Assay text ideally suited for annotation with the CAT

Figure 8. Example of PubChem Assay text ideally suited for annotation with the

CAT. Left: Text from description in PubChem Assay ID 427: yellow = information captured in CAT, green = information not captured but possible for a future version (e.g., controls, data processing), red= information beyond the scope of BAO (technical details) Right: CAT assignments in BioAssay Schema Editor. 
PeerJ Computer Science

Manuscript to b

We have developed a 1536-well cell-based assay for quantitativ against a number of cell lines to determine in vitro cytotoxicity of small molecules. This particular assay uses the Hek 293 cell line which is derived from human embryonic kidney cells (transformed with adenovirus). The CellTiter-Glo luminescent cell viability assay (Promega) is a homogeneous method to measure the number of viable cells in culture. The end point readout of this assay is based on quantitation of intracellular ATP, an indicator of metabolic activity, using the luciferase reaction. Luciferase catalyzes the oxidation of beetle Luciferin to oxyluciferin and light in the presence of ATP. The luminescent signal is proportional to amount of ATP present. Using the CellTiter-Glo luminescent cell viability assay, the amount of cellular ATP was measured in the Hek293 cell line with

40 hours. The assay was pe

1536-well plates. In the screen, tamoxifen and doxorubicin were used as positive controls. Library compounds were measured for their ability to cause acute toxicity in the cell line, as reflected by a decrease in intracellular ATP levels, in a concentration-dependent manner. Data were normalized to the controls for basal activity (DMSO only) and 100\% inhibition (100 uM tamoxifen). AC50 values were determined from concentration-response data modeled with the standard Hill equation.

\section{Key}

Annotated with URI

Added as literal

Not annotated: missed opportunity
PuibChem Assay (ID 427)

Origin: http://pubchem.ncbi.nlm.nih.gov/bioassay/427

bioassay type $\longrightarrow$ bioassay $\longrightarrow$ cell viability assay

assay format $\longrightarrow$ cell based format

assay design method $\longrightarrow$ ATP quantitation using luciferase

assay cell line $\longrightarrow$ is cell line of $\mathrm{HEK} 293$

organism $\stackrel{\text { has organism }}{\longrightarrow}$ Homo sapiens

biological process $\stackrel{\text { has biological process }}{\longrightarrow}$ cell death

has biological macromolecule

target $\longrightarrow$ (not assigned)

assay mode of action $\longrightarrow$ modulation

result $\longrightarrow$ has result $\mathrm{AC50}$

result unit of measurement $\longrightarrow$ (not assigned)

assay screening campaign stage $\longrightarrow$ primary assay

assay footprint $\longrightarrow 1536$ well plate

assay kit $\longrightarrow$ CellTiter-Glo Luminescent Cell Viability Assay

physical detection method $\longrightarrow$ luminescence method

detection instrument $\longrightarrow$ (not assigned)

perturbagen type $\longrightarrow$ compound library 


\section{Figure 9 (on next page)}

Second example of PubChem Assay text ideally suited for annotation with the CAT

\section{Figure 9. Example of PubChem Assay text ideally suited for annotation with the}

CAT. Left: Text from description in PubChem Assay ID 440: yellow = information captured in CAT, pink = information added as 'literal' values (i.e., too specific to exist as a BAO entry, but deemed valuable), green = information not captured but possible for a future version (e.g., controls, data processing), red= information beyond the scope of BAO (technical details). Right: CAT assignments in BioAssay Schema Editor. Annotations added as 'literal' values are highlighted yellow and contained in single quotes. Note that multiple values for a single CAT assignment can be annotated (target biological process, assay mode of action, assay screening campaign stage, perturbagen type). 


\section{Figure $\mathbf{1 0}$ (on next page)}

Example of an assay partially suited for annotation with the CAT

Figure 10. Example of an assay partially suited for annotation with the CAT. Left:

Text from description in PubChem Assay ID 488847: yellow = information captured in CAT, pink = information added as 'literal' values (i.e., too specific to exist as a BAO entry, but deemed valuable), green = information not captured but possible for a future version (e.g., controls, labels of target and ligand, assay quality data $\left.\left(Z^{\prime}\right)\right)$, red= information beyond the scope of BAO (technical details). Right: CATvalues assigned in the BioAssay Schema Editor capture key parameters of the assay yet do not capture the complexity of the assay articulated in the single sentence (arrow): "a flow cytometry protein interaction assay to screen for compounds that compete with RNA binding to GRK2". 
PeerJ Computer Science

A small family of $\mathrm{G}$ protein-coupled receptor (GPCR) kinases (GRKs) negatively regulates heterotrimeric $\mathrm{G}$ protein signaling by phosphorylating multiple sites in the cytoplasmic loops and tails of activated GPCRs [Krupnick, et al. 1998]. Through this process, cells adapt to persistent stimuli that act at GPCRs and protect themselves from damage incurred by sustained signaling. GRKs can also play maladaptive roles in human disease. GRK2 is overexpressed during heart failure, which not only uncouples cardiac receptors from the central nervous system, but also promotes the release of excessive amounts of catecholamines from the adrenal gland [Vatner, et al 1996]. Inhibition of GRK2 by transgenic peptides prevents cardiac failure in mouse models [Rockman, et al. 1998], suggesting that GRK2 is an excellent target for the treatment of heart disease. However, selective small molecule inhibitors of GRKs have not been reported, perhaps due to high homology among the active sites of GRKs and other AGC kinases. Over the last six years, our lab has made significant progress in understanding the structure and function of GRKs, and we are currently investigating the molecular basis for the selective inhibition of GRK2 by a high affinity RNA aptamer [Tse and Boger, 2005].

\section{Preliminary crystallographic studies of this complex demonstrate that the aptamer binds primarily to the \\ RNA aptamer is used in a displacement assay to identify smal \\ molecules that bind to regions on GRK2 \\ tside of its active site that are also critical for activity. This is a}

robust flow cytometry protein interaction assay to screen for compounds that compete with RNA binding to GRK2. Using activity-based secondary screens, we will confirm which hits derived from HTS campaigns exhibit direct binding to GRK2 and inhibit kinase activity. These compounds will be further characterized to establish membrane permeability, their mode of inhibition, and their selectivity for GRK2. Although all active molecules are of interest, small molecules that do not exhibit competitive inhibition with ATP are of particular importance because they would likely represent novel and selective therapeutic leads for the treatment of heart disease.

GRK2 protein is biotinylated using biotinamidohexanoic acid N-hydroxysuccinimide ester(Sigma). The RNA

aptamer is fluorescently labeled on the 3 'end with carboxyfluorescein (

Streptavidin-coated beads (Spherotech) are incubated with biotinylated GRK2 (bGRK2)

\section{concentration of $2 \mathrm{nM}$ for 30 minutes. The BioTek Microf
assay buffer to all but column 1 of a 384 -well assay plate.}

Compounds (10 microM in-well concentration)

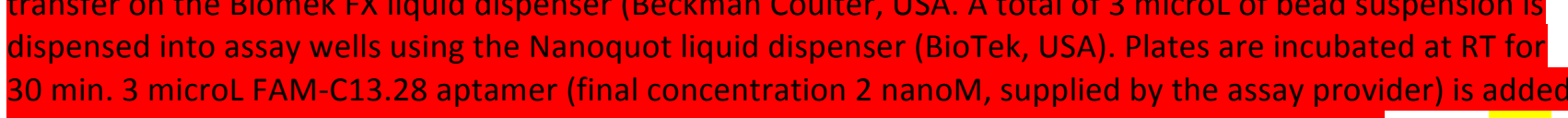

cytometry-based HTS [Kuckuck, et al. 2001] a CyAn flow cytometer (Dako / Beckman Coulter) used to measure the median fluorescence intensity associated

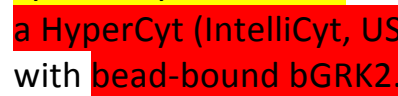

Calculation:

For plates that passed the $Z^{\prime}$ test $\left(Z^{\prime}>.30\right)$ a compound was considered
The $Z^{\prime}$ mean for all the plates was 0.8 with a standard deviation of 0.2 .

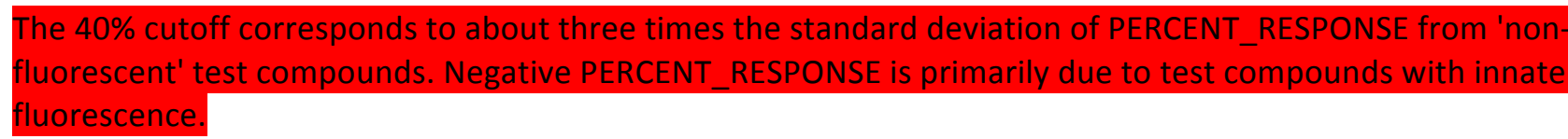

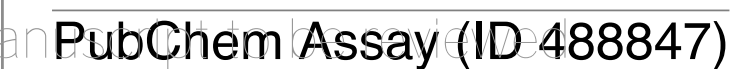

Origin: https://pubchem.ncbi.nlm.nih.gov/bioassay/488847

bioassay type $\longrightarrow$ proassay $\rightarrow$ proin-RNA interaction assay protein-small molecule interaction assay

assay format $\longrightarrow$ biochemical format

has assay design method

assay design method $\longrightarrow$ binding assessment method

assay cell line $\longrightarrow$ is cell line of $\longrightarrow$ (not assigned)

organism $\stackrel{\text { has organism }}{\longrightarrow}$ Homo sapiens

has biological process

biological process $\longrightarrow$ G-protein coupled receptor signaling pathway

has biological macromolecule

"GRK2"

assay mode of action $\longrightarrow$ competitive binding

result $\stackrel{\text { has result }}{\longrightarrow}$ percent response

has unit of measurement
result unit of measurement $\longrightarrow$ percent

assay screening campaign stage $\longrightarrow$ primary assay

assay footprint $\longrightarrow 384$ well plate

assay kit $\longrightarrow$ (not assigned)

has detection method

physical detection method $\longrightarrow$ flow cytometry

detection instrument $\longrightarrow$ CyAn Flow Cytometer

perturbagen type $\longrightarrow$ compound library 


\title{
Dialog box for random lookup of assays from PubChem
}

\author{
Figure 11: Dialog box for random lookup of assays from PubChem.
}

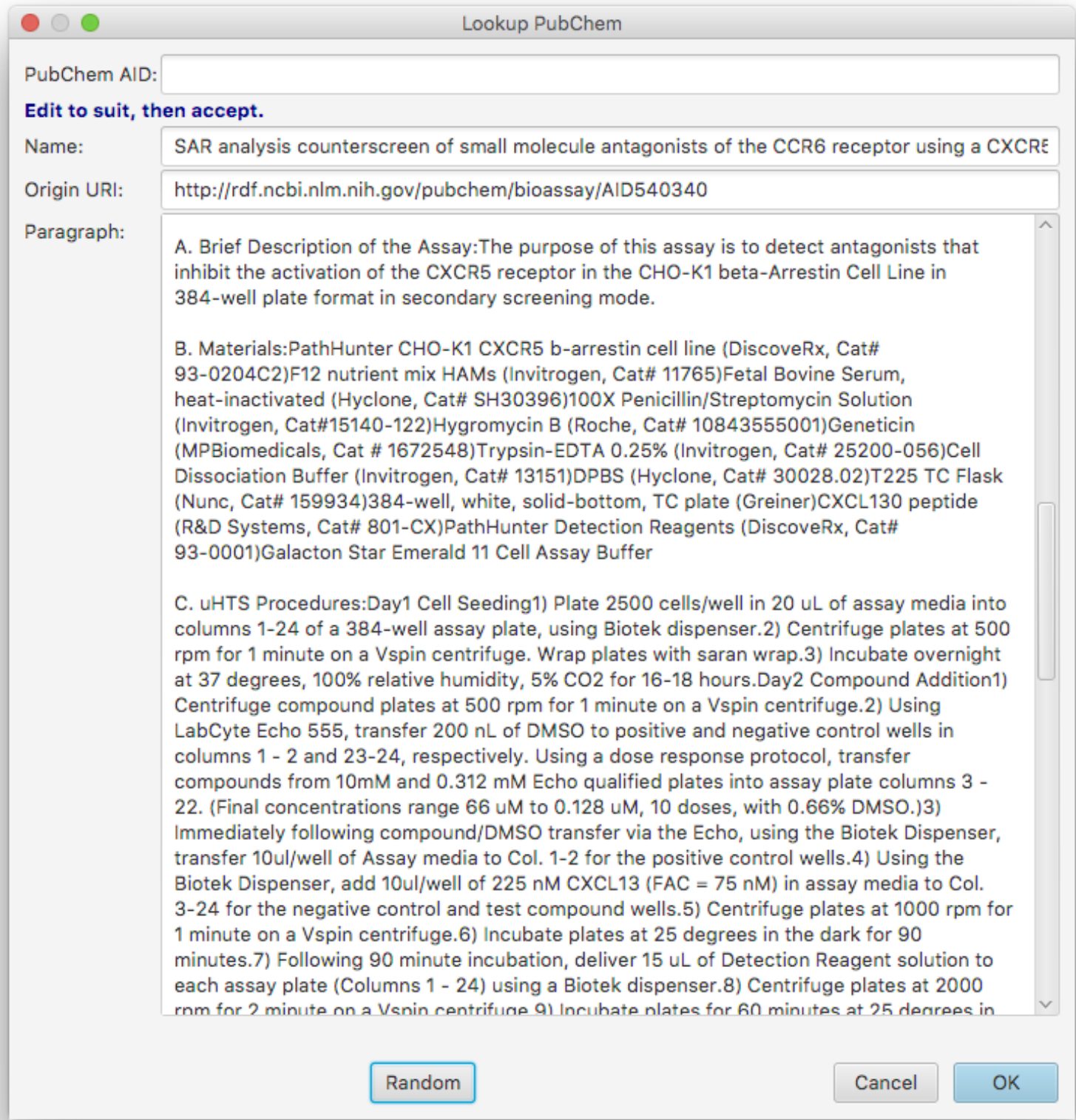




\section{Table $\mathbf{1}$ (on next page)}

Representation of Common Assay Template in Sample Assay Set

Table 1. Representation of Common Assay Template in Sample Assay Set[b] 
Table 1. Representation of Common Assay Template in Sample Assay Set

\begin{tabular}{|l|c|c|}
\hline CAT Assignment & $\begin{array}{c}\text { Test Assays (of 43) } \\
\text { With at Least 1 Value }\end{array}$ & $\begin{array}{c}\text { \# of Unique } \\
\text { Values Annotated }\end{array}$ \\
\hline bioassay type & $43(100 \%)$ & 24 of 88 \\
\hline assay format & $43(100 \%)$ & 6 of 19 \\
\hline assay design method & $43(100 \%)$ & 20 of 76 \\
\hline assay cell line & $24(55.8 \%)$ & 15 of 95 \\
\hline organism & $41(95.3 \%)$ & 11 of 65 \\
\hline biological process & $40(93.0 \%)$ & 28 of 54 \\
\hline target & $32(74.4 \%)$ & 13 of 38 \\
\hline assay mode of action & $43(100 \%)$ & 8 of 13 \\
\hline result & $41(100 \%)$ & 16 of 94 \\
\hline result unit of measurement & $32(74.4 \%)$ & 6 of 56 \\
\hline assay screening campaign stage & $40(93.0 \%)$ & 8 of 23 \\
\hline assay footprint & $36(83.7 \%)$ & 5 of 20 \\
\hline assay kit & $9(20.9 \%)$ & 5 of 93 \\
\hline physical detection method & $42(97.7 \%)$ & 11 of 51 \\
\hline detection instrument & $26(60.5 \%)$ & 9 of 97 \\
\hline perturbagen type & $20(46.5 \%)$ & 3 of 9 \\
\hline
\end{tabular}

